\title{
Acute effects of stretching duration on sprint performance of adolescent football players
}

\author{
Georgia latridou ${ }^{1}$ \\ Yannis Dionyssiotis ${ }^{2}$ \\ Jannis Papathanasiou ${ }^{3}$ \\ Stylianos Kapetanakis ${ }^{4}$ \\ Symeon Galitsanos ${ }^{5}$
}

1 Physical Therapy Unit, Aridaia, Pella; Physical Therapy Unit "Physiospot", Athens, Greece

2 Physical Medicine and Rehabilitation Department, European Interbalkan Medical Center, Thessaloniki, Greece

3 Department of Kinesitherapy, Faculty of Public Health, Medical University of Sofia, Bulgaria; Department of Imaging, Allergology \& Physiotherapy, Medical University of Plovdiv, Bulgaria

4 Spinal Unit Department, European Interbalkan Medical Center of Thessaloniki; Medical School of Alexandroupolis, Democritus University of Thrace, Greece

5 Sports Medicine Department, European Interbalkan Medical Center, Thessaloniki, Greece

Corresponding author:

Yannis Dionyssiotis

Physical Medicine \& Rehabilitation Department,

European Interbalkan Medical Center

10 Asklipeiou str., Pylaia

57001 Thessaloniki, Greece

Tel.: +306946469759

E-mail: yannis_dionyssiotis@hotmail.com

\section{Summary}

Introduction: Athletic performance is the result of the interaction of various factors. The flexibility of the joints plays an important role in athletic performance. The effect of static and dynamic stretching on physical performance has been studied, but with no mention to variable duration. This study aims to examine the effect of duration of acute static and dynamic stretching on sprint performance, in terms of speed and flexibility. Methods: Seventeen football players (mean age $15.9 \pm 0.8$ years) participated in the study. All performed three static stretching protocols and three dynamic stretching protocols with variant duration, in six different training days with random order.
The static and dynamic stretching protocols, lasting 20 seconds each, were performed in three different sets of repetition: 1x20 sec (volume $20 \mathrm{~s}$ ), $2 \times 20 \mathrm{sec}$ (volume $40 \mathrm{~s}$ ) and $3 \times 20 \mathrm{sec}$ (volume $60 \mathrm{~s}$ ). Range of motion was determined during hip flexion, extension and abduction, knee flexion and ankle dorsiflexion using a goniometer. Five pairs of photocells at various distances $(0 \mathrm{~m}, 5 \mathrm{~m}, 10 \mathrm{~m}, 20 \mathrm{~m}$ and $30 \mathrm{~m}$ ) were used for speed evaluation.

Results: Sprint performance remained unchanged at the whole distance of $30 \mathrm{~m}$ after dynamic stretching for 20, 40 and $60 \mathrm{~s}$. Static stretching for 40 and $60 \mathrm{~s}$ the sprint performance decreased $(p<0.05)$, while it remained unchanged for the first 20 meters $(\mathrm{m})$ and decreased in the last $10 \mathrm{~m}$, when the stretching duration was $20 \mathrm{~s}$. Independently from duration static and dynamic stretching increased joint flexibility.

Conclusion: The findings indicate that dynamic stretching does not influence sprint performance, independently of the duration (20-60 s). However, static stretching performed for more than $20 \mathrm{~s}$ (40-60 s) seems to decrease sprint speed. Both static and dynamic stretching improves joint flexibility, in a way irrelevant to duration.

Level of evidence: Ila.

KEY WORDS: static stretching, dynamic stretching, duration, range of motion, sprint performance, football players.

\section{Introduction}

Athletic performance is the result of the interaction of various factors. One of the main factors improving and maximizing performance is the development of physical fitness, which includes physical factors such as strength, power, speed and flexibility, as well as their combinations such as strength and muscular endurance. The flexibility of the joints plays an important role in athletic performance ${ }^{1}$.

Several studies have been published regarding the direct effect of stretching on power production and jumping. However, the effect of stretching on free running speed of athletes has not been extensively studied. Most studies report a negative effect of acute stretching on power and jump height, while the outcome on running speed is contradictory, maybe due to the intervention of other variables related to the 
performance, such as output power, contraction speed, running economy and psychology of the individual athlete 2-5. $^{2-}$

Siatras et al. examined the direct effect of static and dynamic acute stretching on gymnasts' free running speed in vaulting. Participants performed three programs. In the first one they performed 5 min general warm-up and static stretching in the legs, in the second program they executed 5 min general warm and active stretches and in the third program they executed only general warm-up $5 \mathrm{~min}$. The results showed a significant decrease in the performance when static stretching was preceded, while no inhibitory effect was observed with active stretching ${ }^{6}$. Fletcher and Jones examined the effect of four different stretching methods on the performance of free running speed to amateur rugby athletes. The first method included static stretching for $20 \mathrm{sec}$ (passive static method). The second method consisted of slow rhythmic movements and displacements in the joint for $20 \mathrm{sec}$ (active static method), the third method included stretching, which were rhythmically with the contraction of the agonists and active lengthening of antagonists (active dynamic method) and the fourth method, in which the practitioner performed 20 repetitions of the static position with stretching exercises similar to the active motion (static dynamic method). The researchers found that static stretching had a negative impact on the performance of free running speed at 20 meters in rugby athletes, while performance in running speed was increased or not affected with dynamic stretching7 ${ }^{7}$. Bacurau et al. demonstrated that static stretching on lower extremities results in reduced maximum muscle strength, despite the acute favorable effect on flexibility compared to ballistic stretching ${ }^{8}$. Nelson et al. also examined the direct effects of static stretching on performance of free running speed in professional athletes of various sports. The program of static stretching had duration of $120 \mathrm{sec}$ (4×30"). They found that static stretching had a negative impact on the performance of athletes ${ }^{9}$. However, Kokkonen et al. measured an improvement in flexibility and $20 \mathrm{~m}$ sprint performance after the application of a chronic 10-week intervention program of static stretching of lower extremities ${ }^{10}$. It is clear that acute stretching negatively affects sprint performance ${ }^{6-9}$, while a chronic static stretching protocol maybe beneficial ${ }^{10}$.

Considering the fact that the aforementioned stretching protocols have duration of $45 \mathrm{sec}$ up to 1 hour for each muscle group and, though, are not used i.e. by football players before their sport activity ${ }^{11,12}$, it would be of interest to examine whether the duration of stretching plays a role in the performance of free running speed. The effect of static and dynamic stretching on physical performance has been studied, but with no mention to variable duration. The innovation of study lies on the parameter of duration. The purpose of this study is to examine the direct effect of duration of static and dynamic stretching protocols on (I) free running speed performance and (II) joints flexibility in 17 young amateur football players.

\section{Materials and methods}

Seventeen (17) young football players aged 15.9 \pm 0.8 years participated in this study. All athletes were agreed to participate voluntary in the study and gave oral consent. The research protocol was designed according to the Declaration of Helsinki and was conducted in compliance with the Aristotle University of Thessaloniki code of conduct.

\section{Flexibility measurements}

For flexibility measurements, two types of goniometers were used: a) the goniometer Brodin; is only used in the abduction of the hip and consists of a fixed and a movable arm projecting by $60 \mathrm{~cm}$, in order to adapt on the longitudinal anatomical axis of the femur and b) the goniometer Myrin (Lic Rehab 17183), which measures vertically and horizontally and was used in other joints; its function is based on the bending goniometer Leighton (1955): it consists of a circular scale (0-180) with a turntable and two indicators. The one indicator is arranged in the center of the disc and is controlled by gravity, while the other is orientation indicator for horizontal movements. Measurements of the flexibility of the joints were expressed in degrees.

Measurements of flexibility included hip extension, flexion and abduction, knee flexion and ankle dorsiflexion with the knee in a flexed position, as suggested by Ekstrand et al. ${ }^{13}$ All measurements were performed only on the participants' right leg. During each training session flexibility measurements were performed at two time points: one before the initiation of the training program and one after the program was completed. The flexibility measurements were performed by two testers, experienced and familiar with the technique, on a medical bed - except the ankle measurement which was performed at the stand-up position with the knee flexed on a low and flat object. Examiners gave preliminary instructions to the participants about measurements, the duration of the measurement and its details. The points of each joint were marked by the same examiner always. Maximum passive joint movement was assessed by the examiner measuring the maximum joint resistance developed by the participant's muscle group stretched. There was no intermission between each joint measurement, except only the time necessary for the correct placement of the instrument on each joint. All measurements were performed under the same conditions for all participants. The results of each test of the measurement were recorded in a special data acquisition protocol.

\section{Stretching protocol}

Football players were applied three static and dynamic stretching programs of different duration, which abstained one week from each other. The series, the manner and the time executed were common for all players. Each person was performing the particular program on different days, in order to avoid possible 
adjustments or fatigue during measurements. The protocol included: I. initial flexibility measurements; II. 10 min warm-up; III. initial (i.e. baseline) maximum speed measurement, IV. application of the stretching program; V. final flexibility and maximum speed measurements. The first program included static stretching with duration $20 \mathrm{sec}(1 \times 20)$. The second program involved the same procedure, except that the stretching program was performed 2 times $(2 \times 20)$ for 20 sec. The third program was exactly the same, except that the stretching program was performed 3 times $(3 \times 20)$ for $20 \mathrm{sec}$. These 3 programs of static stretching were executed by the same process, but not with the same duration. In the 4th program the duration length of dynamic stretching was $20 \mathrm{sec}$ and was executed only once $(1 \times 20)$. The 5 th program was performed twice $(2 \times 20)$ for $20 \mathrm{sec}$, while in the 6 th was performed three times $(3 \times 20)$ for $20 \mathrm{sec}$. The stretching programs included stretches so as to have anterior (quadriceps) femoris muscles, posterior femoris muscles, posterior tibial muscles, iliopsoas muscle and adductor muscles stretched. Each stretching was performed in two parts of the body alternately. The rest period between repetitions lasted $20 \mathrm{sec}$. The repetitions were completed for each specific muscle group and then the next muscle group was taken on. Stretching exercises selected were performed in the same order, manner and time in all the flexibility programs.

\section{Free running speed measurement protocol}

The running speed of athletes was measured by photocells of company TAG HEUER with integrated printer in electronic timer Omega, capable of measuring up to milliseconds. For the free running speed measurements, the electronic timer was connected with five pairs of photocells. The photocells were mounted in a special tripod at shoulder height. The time was recorded each time the trainee interrupted the photocell beam with his shoulder. We assessed the maximum speed at distances $0 \mathrm{~m}, 5 \mathrm{~m}, 10 \mathrm{~m}, 20 \mathrm{~m}$ and $30 \mathrm{~m}^{14}$.

At each training session, two sprints were performed at baseline, before the stretching program, and two sprints at the end of the stretching program. All sprints were performed at maximum intensity.

Initially the subjects performed a 10 min general and specific preheating ${ }^{7}$. Then, two sprints with maximal intensity were performed and followed by the intervention program of stretching. Finally, the participants performed two final sprints. Of the two attempts performed in each test, the best one was recorded (i.e. the shortest time). The intermission period between the two sprints was 3 min and between the two initial and final sprints was around 20 min (the whole period of the intervention including stretching program 10-12 min and final flexibility measurements). Sprint started from an upright position, with the dominant foot in front of the other and one meter right behind the imaginary line of first photocells. All participants were wearing football shoes and provided verbal stimula- tion. The free running speed measurements were carried out in a football stadium. The conditions under which all measurements were performed were the same for all athletes (no sport activity prior and the day of the measurement).

\section{Statistical analysis}

There were two independent variables, the variable $A$ (training program) with six levels and the variable $B$ (initial measurements) with two levels. The dependent variables were two: I. flexibility after the training program; II. free running speed after the implementation of training programs (final measurements). For the statistical analysis the variance analysis method $3 \times 2$ (programs $\times$ measurement) (ANOVA) was used, with the last factor to be repeated in order to compare the significance of the difference of averages between values in the flexibility and free running speed among the 3 programs of static stretching. The same was used also for the 3 programs of active stretching. To detect the difference between the initial and final values of flexibility and free running speed method ttest for independent measurements for each stretching program separately was used. The statistical significant level was defined $p<0.05$.

\section{Results}

The statistical analysis showed significant improvements $(p<0.05)$ in the kinematic range of joints after running any protocol, either it was dynamic or static type. Concerning the running speed, the statistical analysis showed a significant reduction in free running speed of performance in every distance traveled by the adolescents players $(0-5,0-10,0-20,0-30 \mathrm{~m})$, when static stretching was applied of duration $2 \times 20$ and $3 \times 20$ sec (Fig. 1).

When the duration of static stretching was only 20 sec (Fig. 2), a reduction in the running speed of young players was observed only in the last few meters of the total distance of $30 \mathrm{~m}(\mathrm{p}<0.05)$.

On the contrary, no significant decrease in performance in the other intervals of the total distance of 20 $\mathrm{m}(0-5,0-10$ and $0-20 \mathrm{~m})$ was observed $(\mathrm{p}>0.05)$. The performance of free running speed remained at the same level across all distances traveled by the adolescent football players (0-5, 0-10, 0-20, 0-30 m) independently of the duration of dynamic stretching once for $20 \mathrm{sec}(1 \times 20)$, twice for $20 \mathrm{sec}(2 \times 20)$ or three times for $20 \mathrm{sec}(3 \times 20)$ (Fig. 3), (p>.0.05).

\section{Discussion}

Research is mainly focused on the direct effect of muscle stretching in muscular performance of athletes when applied during the warm-up. This study examined the direct effect of duration of static and dynamic stretching in flexibility and free running speed performance in adolescent football players. 


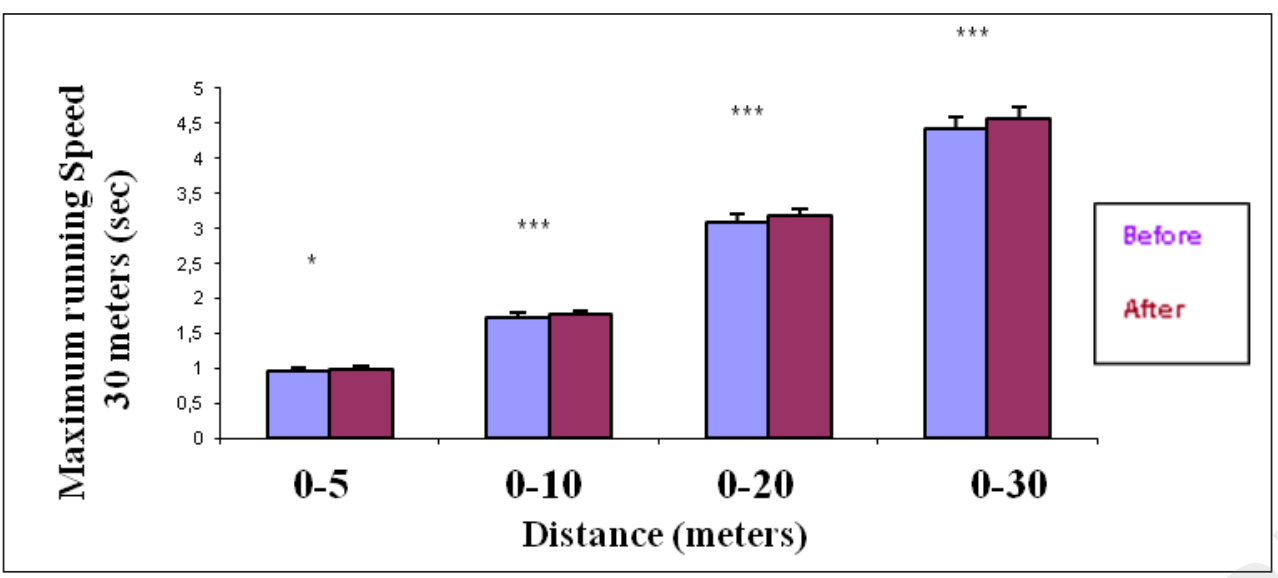

Figure 1. Significant reduction in free running speed of performance in every distance traveled by the adolescents players $(0-5,0-10,0-20,0-30 \mathrm{~m})$, when static stretching was applied of duration $2 \times 20$ before and after the application of the protocol. Mean values $\pm S D$; * and ${ }^{* * *}$ represent statistical values of $p<0.05$ and $p<0.01$, respectively. Similar results are depicted in a graph (not shown here) when static stretching was applied of duration $3 \times 20$ sec.

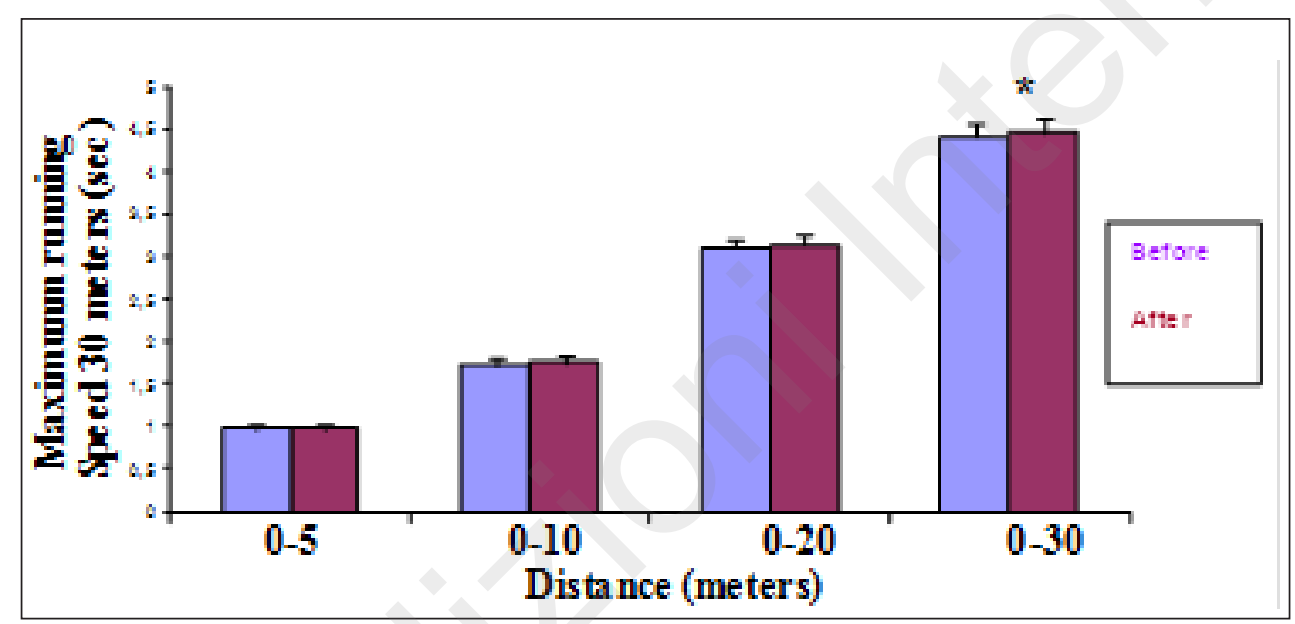

Figure 2. Within a $20 \mathrm{sec}$ duration of static stretching significant reduction in the running speed of adolescent players was only observed in the last meters of the total distance of $30 \mathrm{~m}(\mathrm{p}<0.05)$ before and after the application of the protocol. No significant difference in maximum running speed in the other intervals of the total distance of $20 \mathrm{~m}(0-5,0-10$ and $0-20 \mathrm{~m})$ was observed. Mean values $\pm S D$; * represents statistical value $p<0.05$.

The results showed improvements in joint range of motion of the legs after application of either static or dynamic stretching, regardless of their duration. Our results also showed significant reductions in the performance of free running speed when the total duration of static stretching was 40 and $60 \mathrm{sec}$, while it remained unchanged for the first 20 meters, when the duration of stretching was $20 \mathrm{sec}$ only. On the contrary, running speed was not affected when dynamic stretching was preceded, independently of the duration $(20,40$ or $60 \mathrm{sec})$.

Our results agree with studies performed long duration programs with static or dynamic or active method $^{15-19}$. Flexibility of the joints is improved as the muscle lengthens statically or dynamically. Older reports have shown that the muscle is adjusted depending on the elongation ${ }^{20}$. Zakas et al. investigated the acute effects of passive stretching after a general warming-up bout, as well as the effects of passive stretching alone of the leg muscles, by the application of 3 different flexibility-training protocols in separate training sessions in 18 young footballers. The first protocol included jogging for 20 minutes; the second consisted of the same followed by passive stretching of the lower extremities and the trunk; the third included passive stretching alone. The results showed that both stretching exercises alone and stretching exercises after preheating were of great importance to improve flexibility of all the joints of the lower limbs and the trunk flexion ${ }^{19}$.

Studies comparing the effect of dynamic or static stretching on muscle performance are limited and not considering the role of duration of stretching on muscle performance. Our research is focusing on how 


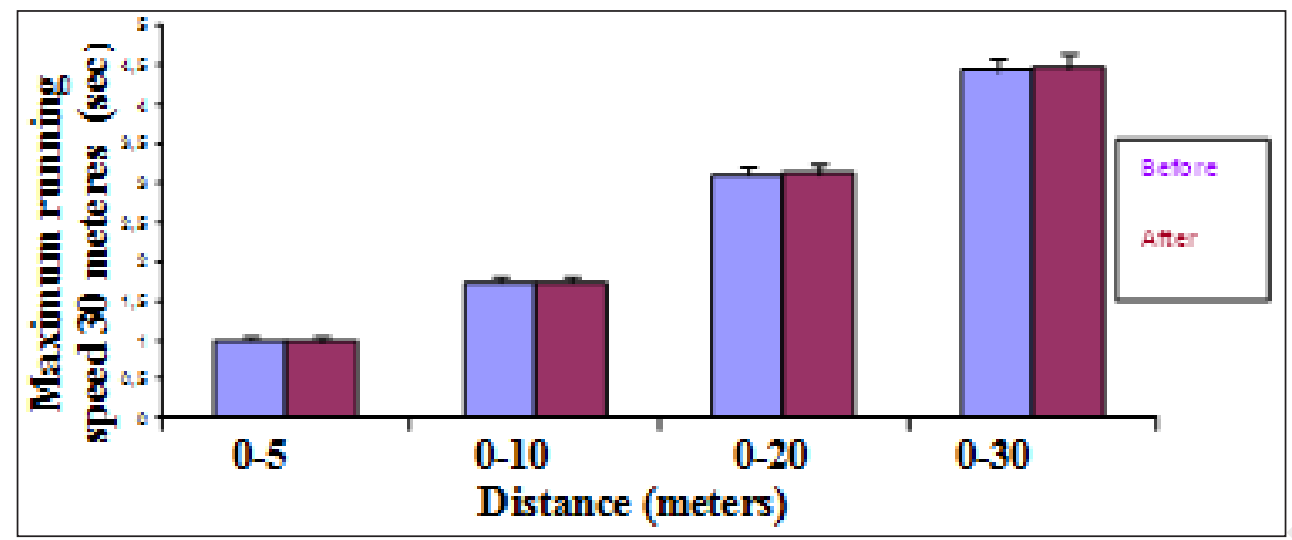

Figure 3. Maximum running speed remained at the same level across all distances traveled by the adolescent players (0-5, $0-10,0-20,0-30 \mathrm{~m})$ independently and non significant of the duration of dynamic stretching once for $20 \mathrm{sec}(1 \times 20)(\mathrm{graph}$ not shown), twice for $20 \mathrm{sec}$ (graph not shown) and three times for $20 \mathrm{sec}(3 \times 20)$ (Fig. 3), (p>.0.05) before and after the application of the protocol. Mean values \pm SD.

the duration of stretching exercises in a training period affects the flexibility of the joints and the running speed of football players. Regarding the direct effect of stretching on performance of free running speed our results agree with research using long duration, at least over $20 \mathrm{sec}$, and with similar research of static stretching type assets executed ${ }^{6,21}$. Siatras et al. found a significant decrease in the performance of free running speed when a program of static stretching duration $60 \mathrm{sec}(2 \times 30)$ was applied. Reduction in free running speed was found in our results, when static stretching either $40(2 \times 20)$ or $60(3 \times 20)$ sec was applied which suggests that a static stretching duration over $20 \mathrm{sec}$ can have a negative influence on free running speed, while a duration of $20 \mathrm{sec}$ does not adversely affect the running speed at the first $20 \mathrm{~m}$. The results concerning that a static stretch duration lasting more than $20 \mathrm{sec}$ is reducing free running speed stand in line with the results of Nelson et al. who found reductions in free running speed in professional athletes, when static stretching $120 \mathrm{sec}$ $(4 \times 30)$ was performed ${ }^{21}$. Unlike static stretching, the results of this study showed running speed not to be negatively impacted when either short $(20 \mathrm{sec})$ or longer (40 or $60 \mathrm{sec}$ ) dynamic stretch was performed. The results of this study are consistent with results from other studies, where the duration of active stretching was $60 \mathrm{sec}$ or $20 \mathrm{sec}^{6,7}$. These results suggest that dynamic stretching could not adversely affect running speed independently of the duration.

The mechanisms that are held responsible for reductions in muscle performance after static stretching are not yet specified, and as possible explanations have been proposed mechanical factors related to the muscle-tendon system ${ }^{22-25}$, the neural inhibition 25,26 and deterioration of muscle tissue of prolonged static stretching, as evidenced by increased creatine kinase detected in the blood ${ }^{27}$. Static stretching possibly increases the compliance of the muscles and tendons units and, consequently, reduces their capability as elastic energy storage ${ }^{7}$. Moreover, dilatation over $20 \%$ of the length of the muscle fibers may damage the contractile elements with a direct negative effect on power output.

The reason why dynamic stretching does not adversely affect the muscular performance remains unclear. One of the possible mechanisms is the largest increase in the internal temperature of the body, induced by active stretching compared to static stretching. The increase in the internal temperature causes an increase in the sensitivity of nerve receptors and increase the speed of nerve conduction, facilitating muscle contractions ${ }^{28}$. Some researchers, however, deny that the internal differences in temperature can cause changes in the performance of free running speed ${ }^{7}$. It is proposed that rhythmical movements performed in active stretching protocols better simulate athletic activity. Probably dynamic stretching enhances proprioception, movement coordination and allows the muscles to stimulate earlier and faster than static stretching, leading in the production of more power explaining the insignificant differences in the sprint time ${ }^{7,29}$.

\section{Conclusion}

Flexibility of hip, knee and ankle joints was improved in adolescent football players after static or dynamic stretching exercises, independent of their duration. Static stretching of short duration did not adversely affect the running speed up to $20 \mathrm{~m}$. However, longer (40 or $60 \mathrm{sec}$ ) duration static stretching increases sprint time over the distance of $20 \mathrm{~m}$. Active stretching exercises do not alter running speed independent of their duration. We conclude that stretching duration is a determining factor on the outcome of static stretching on sprint performance, while it does not seem to affect the outcome of active stretching. Improvement of joint flexibility after stretching (active or passive) is not influenced by duration, as well. 


\section{Ethics}

The Authors declare that this research was conducted following basic ethical aspects and international standards as required by the journal and recently update in ${ }^{30}$.

\section{References}

1. Weineck J. Optimales Fussballtraining: Das Konditionstraining des Fussballspielers. Spitta Verlag GmbH \& Co. 1992.

2. Kruse N, Barr M, Gilders R, Kushnick M, Rana S. Effect of different stretching strategies on the kinetics of vertical jumping in female volleyball athletes. Journal of Sport and Health Science. 2015;4:364-370.

3. Makaruk H, Makaruk B, Kedra S. Effects of warm-up stretching exercises on sprint performance. Physical Education and Sport. 2008;52:23-26.

4. Saoulidis J, Yiannakos A, Galazoulas C, Zaggelidis G, Armatas V. Acute Effect of Short Passive and Dynamic Stretching on $20 \mathrm{~m}$ Sprint Performance in Handball Players. Physical Training. 2010 Aug. http://ejmas.com/pt/2010pt/ptart_saoulidis_1009.html

5. Shrier I. Does stretching improve performance? A systematic and critical review of the literature. Clinical Journal of Sport Medicine. 2004;14:267-273.

6. Siatras T, Papadopoulos G, Mameletzi D, Gerodimos V, Kellis S. Static and Dynamic Acute Stretching Effect on Gymnasts' Speed in Vaulting. Pediatric Exercise Science. 2003;15:383391.

7. Fletcher IM, Jones B. The effect of different warm up stretch protocols on 20 meter sprint performance in trained rugby union players. The Journal of Strength and Conditioning Research. 2004;18:885-888.

8. Bacurau RFP, Monteiro GDA, Ugrinowitsch C, Tricoli V, Cabral LF, Aoki MS. Acute effect of a ballistic and static stretching exercise bout on flexibility and maximal strength. Journal of Strength and Conditioning Research. 2009;23:304308.

9. Nelson AG, Driscoll NM, Landin DK, Young MA. Schexnayder IC. Acute effects of passive muscle stretching on sprint performance. Journal of Sports Sciences. 2005;23:449-454.

10. Kokkonen J, Nelson AG, Eldredge C, Winchester JB. Chronic Static Stretching Improves Exercise Performance. Medicine Science of Sports and Exercise. 2006;39:1825-1831.

11. Young W, Elliott S. Acute Effects of Static Stretching, Proprioceptive Neuromuscular Facilitation Stretching, and Maximum Voluntary Contractions on Explosive Force Production and Jumping Performance. Research Quarterly for Exercise and Sport. 2001;172:273-279.

12. Avela J, Kyrolainen $\mathrm{H}$, Komi PV. Altered reflex sensitivity after repeated and prolonged passive muscle stretching. Journal of Applied Physiology. 1999;86:1283-1291.

13. Ekstrand J, Wiktorsson M, Oberg B, Gillquist J. Lower extremity goniometric measurements: A study to determine their reli- ability. Archives of Physical Medicine and Rehabilitation. 1982;63:171-175.

14. Kolath, E, Quade K. Measurement of sprinting speed of professional and amateur soccer players. In Reilly $\mathrm{T}$, Clarrys $\mathrm{J}$ Stibbe A, editors. Science and football II. London: E \& FN Spon. 1993:1-36.

15. Zakas A, Galazoulas C, Grammatikopoulou M, Vergou A. Effects of stretching exercise during strength training in prepubertal, pubertal and adolescent boys. Journal of Bodywork and Movement Therapies. 2002;6:170-176.

16. Fredrick GA, Szymanski DJ. Baseball (part I): dynamic flexibility. Strength Conditioning Journal. 2001;23:21-30.

17. Hedrick A. Dynamic flexibility training. Strength Conditioning Journal. 2000;22:33-38.

18. Madding SW, Wong JG, Hallum A. Effects of duration or passive stretching on hip abduction range of motion. The Journal of Orthopaedic and Sports Physical Therapy. 1987:8:409-416.

19. Zakas A, Grammatikopoulou MG, Zakas N, Zahariadis P, Vamvakoudis $E$. The effect of active warm-up and stretching on the flexibility of adolescent soccer players. J Sports Med Phys Fitness. 2006;46:57-61.

20. Van Der Poel G. The science of conditioning. Flexibility. In: Verheijen R, editor. The complete handbook of conditioning for soccer. Reedswain: Spring. 1998:54-56.

21. Nelson AG, Driscoll NM, Landin DK, Young MA, Schexnayder IC. Acute effects of passive muscle stretching on sprint performance. Journal of Sports Sciences. 2005;23:449-454.

22. Cornwell A, Nelson A, Heise GD, Sidaway B. Acute effects of passive muscle stretching on vertical jump performance. Journal of Human Movement Studies. 2001;40:307-324.

23. Kokkonen J, Nelson AG, Cornwell A. Acute muscle stretching inhibits maximal strength performance. Research Quarterly for Exercise and Sport. 1998;69:411-415.

24. Nelson AG, Kokkonen J. Acute ballistic Muscle Stretching Inhibits Maximal Strength Performance. Research Quarterly for Exercise and Sport. 2001;72:415-419.

25. Behm D, Button D, Butt J. Factors affecting force loss with prolonged stretching. Canadian Journal of Applied Physiology. 2001;126:262-272.

26. Fowles JR, Sale DG, Mac Dougall JD. Reduced strength after passive stretch of the human plantarflexors. Journal of Applied Physiology. 2000;89:1179-1188.

27. Smith LL, Brunetz MH, Chenier TC, McCammon MR, Houmard JA, Franklin ME, Israel RG. The effects of static and ballistic stretching on delayed-onset muscle soreness and creatine kinase. Research Quarterly for Exercise and Sport. 1993;64:103-107.

28. Shellock FG, Prentice WE. Warming-up and stretching for improved physical performance and prevention of sports-related injuries. Sports Medicine. 1985;2:267-278.

29. Fletcher IM, Anness R. The acute effects of combined static and dynamic stretch protocols on fifthy-meter sprint performance in track-and-field athletes. J Strength Cond Res. 2007;21:784-787.

30. Padulo J, Oliva F, Frizziero A, Maffulli N. Muscles, Ligaments and Tendons Journal - Basic principles and recommendations in clinical and field science research: 2016 update. MLTJ. 2016;6(1):1-5. 